\title{
Entropy-Based Non-Data-Aided SNR Estimation
}

\author{
Ferran de Cabrera, Student Member, IEEE and Jaume Riba, Senior Member, IEEE \\ Signal Theory and Communications Department, Technical University of Catalonia (SPCOM/UPC) \\ \{ferran.de.cabrera, jaume.riba\}@upc.edu
}

\begin{abstract}
A non-data-aided SNR estimation of a communication channel is addressed in this paper. The main contribution relies on the relationship between an entropy measure of a given constellation and the link quality. The main advantage is that the second order Rényi entropy is invariant to the rotation of the constellation and it is not envelope-based as the moment-based methods, enabling to asymptotically approximate the CRLB of coherent estimators at high SNR for an increasing data size. Comparative results are shown with other non-data-aided SNR estimation techniques.
\end{abstract}

Index Terms-SNR estimation, Rényi entropy, Information potential, Kernel methods, Digital communications.

\section{INTRODUCTION}

The assessment of the link quality of a channel is an important issue in many wireless communication systems. The Signal to Noise Ratio (SNR) is a measure that quantifies this quality and its estimation from the received signal is crucial for many fields of digital communications such as optimum signal detection or adaptive coding and modulation. Generally speaking, Maximum Likelihood (ML) schemes are adopted in Data-Aided (DA) estimation, where the transmitted sequence of symbols is known ([15] and references therein). In this case, the asymptotic convergence to the Cramér-Rao Lower Bound (CRLB) is ensured, thus providing an efficient estimator. However, in Non-Data-Aided (NDA) estimation it is assumed that the sequence of symbols transmitted is unknown, ultimately causing the ML estimator to lack closedform expressions.

In order to seek alternatives for the NDA problem, some estimators were proposed based on the relationship between even-numbered moments. Although many of the algorithms that have been proposed over the years present different performances that depend on the block length of the transmitted sequence, the actual value of SNR or the number of symbols in a given constellation, the moment-based method presents the more robust solution, particularly for low to medium SNR range [11]. The purpose is to gain invariance to scale, phase and frequency errors, providing a consistent solution for Constant Modulus (CM) constellations. The $M_{2} M_{4}$ approach exemplifies this path by measuring the second and fourth order statistics [3], [10]. However, for non-CM constellations the $M_{2} M_{4}$ algorithm performance at high SNR is degraded. To cope with this problem, some algorithms based on higher order statistics have been proposed, which can improve the

This work is supported by project TEC2016-76409-C2-1-R (WINTER), "Ministerio de Economia y Competividad", Spanish National Research Plan, by the Catalan administration (AGAUR) under 2017-SGR-578, and fellowship FI 2019 by the Secretary for University and Research of the "Generalitat de Catalunya" and the European Social Fund. performance of the $M_{2} M_{4}$ estimator for relatively higher values of SNR [1]. As a drawback, moment-based estimators consider the worst-case scenario with respect to phase and frequency errors, since they are based on the envelope of the received signal. This fact results in a CRLB that is asymptotically, in terms of increasing SNR, two times greater than the coherent case [8] that is benefiting from both phase and modulus.

Conversely, some alternatives have been proposed that seek for other indicators of the SNR, usually by combining second and fourth order statistics with other techniques. For instance, in [8] a combination of the $M_{2} M_{4}$ and the Expectation Maximization (EM) algorithm is proposed, although it is entirely based on the envelope-based approach. On the other hand, the author in [4] proposes an EM algorithm that seeks to perform as close as possible to the coherent CRLB, although its computational complexity greatly depends on the steps taken by the algorithm and it can carry an initialization problem if the received signal presents phase errors. In [14] the estimation is built from second order statistics and the cyclostationarity of the signal. In this case, instead of measuring the SNR at the output of the matched filter assuming optimal sampling instants, the signal is evaluated before the matched filter with oversampling, exploiting the cyclostationarity, but with varying performances depending on the roll-off factor of the bandwidth in the frequency domain. More recently, in [6] the estimation is based on the Kolmogorov-Smirnov test between the empirical and the pre-stored cumulative distribution functions, and although the performance is improved for a wide SNR range, it requires to store all the reference functions and to compare them until a match is found.

In this paper, we propose to estimate the SNR based on an estimation of an information-theoretic measure. We start from the observation that the second order Rényi entropy of a given constellation can be expressed solely through its signal and noise power and it is invariant to rotations. Therefore, by estimating the Rényi entropy first we can measure the SNR on a second stage, obtaining an estimate that is phase invariant. Furthermore, at high SNR values we propose to combine this entropy measure with the second order moment, which eases the computational complexity and tends asymptotically to the coherent CRLB for an increasing observation interval length.

\section{RÉNYI ENTROPY OF A NOISY CONSTELLATION}

Consider a constellation $\mathcal{C}$ composed by $M$ complex zeromean symbols $\left\{s_{m}\right\}_{m=0, \ldots, M-1}=r_{m} e^{j \theta_{m}}$ with unit variance $E\left\{\left|s_{m}\right|^{2}\right\}=1$. The equivalent baseband model is the following

$$
x_{k}=\sqrt{S} s_{k}+\sqrt{N} w_{k},
$$


with $x_{k}$ independent and identically distributed (i.i.d.) observations, $S$ and $N$ the signal and noise powers, respectively, and $w_{k}$ a sample of a complex zero-mean Gaussian process with unit variance. Therefore, the Signal to Noise ratio (SNR) is defined by $\rho=S / N$. Assuming all symbols are equally probable, we can express the Probability Density Function (PDF) of the constellation for a known $S$ and $N$ as a Gaussian Mixture Model (GMM) with

$$
f_{X}(x)=\frac{1}{M} \sum_{m=0}^{M-1} \mathcal{C N}\left(\sqrt{S} s_{m}, N\right),
$$

being $\mathcal{C} \mathcal{N}\left(\mu, \sigma^{2}\right)$ a complex Gaussian distribution with mean $\mu$ and variance $\sigma^{2}$.

The second order Rényi entropy [13] of a continuous random variable with $\mathrm{PDF} f_{X}(x)$ is defined as

$$
H_{2}(X)=-\ln \left(\int f_{X}^{2}(x) d x\right)=-\ln (V(X)),
$$

being $V(X)$ the expected value of the PDF, which corresponds to the Information Potential (IP) [12]. Given $K$ complex i.i.d. samples $\boldsymbol{x}=\left\{x_{k}\right\}_{k=0, \ldots, K-1}$ an estimator of the IP, and consequently of the Rényi entropy, can be defined by the Parzen window method [12], which is based on a plug-in estimate of the PDF. If Gaussian kernels are used, the estimate simplifies to the following expression:

$$
\hat{V}_{v}(\boldsymbol{x})=\frac{1}{K^{2}} \sum_{i=0}^{K-1} \sum_{j=0}^{K-1} g_{v}\left(x_{i}-x_{j}\right),
$$

being the kernel

$$
g_{v}(\lambda)=\frac{1}{2 \pi v} \exp \left(-\frac{|\lambda|^{2}}{2 v}\right)
$$

with $v$ the kernel bandwidth and $|\lambda|$ indicating the modulus of the complex number $\lambda$. Since $\hat{V}_{v}$ is a biased estimator with $\hat{V}_{v}=\frac{1}{K} \frac{1}{2 \pi v}+\hat{U}_{v}$, we will define the U-statistics

$$
\hat{U}_{v}(\boldsymbol{x})=\frac{2}{K(K-1)} \sum_{0 \leq i<j \leq K-1} \sum_{v} g_{v}\left(x_{i}-x_{j}\right),
$$

which is an unbiased estimate of the IP [5].

On the other side, the IP of a given constellation $\mathcal{C}$ can be evaluated by the integral in (3) using (2), leading to a closedform expression for any constellation. Alternatively, we can easily obtain the IP following (4) but, instead of observations, by means of the constellation symbols:

$$
\begin{aligned}
V_{\mathcal{C}}(S, N) & =\frac{1}{K^{2}} \sum_{i=0}^{M-1} \sum_{j=0}^{M-1} g_{N}\left(\sqrt{S} s_{i}-\sqrt{S} s_{j}\right) \\
& =\frac{1}{K^{2}} \frac{1}{2 \pi N} \sum_{i=0}^{M-1} \sum_{j=0}^{M-1} \exp \left(-\frac{S\left|s_{i}-s_{j}\right|^{2}}{2 N}\right)
\end{aligned}
$$

This expression ensures that the IP can be expressed solely by $S$ and $N$ for a known number of symbols $M$, and consequently we can ensure that the SNR and the IP can be related. It is worth mentioning that we are using a double variance notation in the kernel denominator in equations (5) and (7), instead of using solely $v$ and $N$ respectively. This is due to equalize the kernel bandwidth to the real variance of the symbols being processed, that is the double in the case of the subtraction between symbols, and equivalently within equation (5).

One of the most important aspects of (7) is that the expression can be further simplified when the actual SNR is high enough. If that is the case, the entropy can be expressed solely by the number of symbols $M$ and the noise power $N$ with

$$
\lim _{S N R \rightarrow \infty} V_{\mathcal{C}}=\frac{1}{M 2 \pi N},
$$

and it is approximately achieved when there is no significant overlap between components of the GMM in (2). This expression eases the estimation of high SNR values since the IP function becomes linear in terms of the SNR, an advantage that will be used in Section III.

Additionally, note that under Gaussian assumption for both kernel and data, the expectation of the estimator $\hat{U}_{v}(\boldsymbol{x})$ does not correspond to the true IP but a perturbed version caused by a non-zero kernel bandwidth $v$. The insight is that for every observation that is further measured through a Gaussian kernel, we are actually convoluting the kernel $g$ with the actual PDF. The result is then another Gaussian function with the variance being the sum of the kernel bandwidth and the variance of the components of the GMM. Hence the following relation holds:

$$
E\left\{\hat{U}_{v}(\boldsymbol{x})\right\}=V_{\mathcal{C}}(S, N+v),
$$

which is the main rationale behind this work and it will used as the principal tool for the estimator.

The main advantage of this method is that, for a given phase error $\phi$ conveyed from the symbols of the constellation as $\left\{s_{m}\right\}_{m=0, \ldots, M-1}=r_{m} e^{j \theta_{m}+\phi}$, we can state that the IP of the constellation is invariant to this error:

$$
\left.V_{\mathcal{C}}(S, N)\right|_{\boldsymbol{r} e^{j \boldsymbol{\theta}}}=\left.V_{\mathcal{C}}(S, N)\right|_{\boldsymbol{r} e^{j \boldsymbol{\theta}+\phi}} .
$$

Therefore, the phase error does not need to be estimated nor it needs to be corrected. However, we should not equate this phase error robustness with the one from the momentbased method. While we rely on the invariant property of the Rényi entropy to rotations, the moment-based method relies on measuring the envelope of the signal received, thus suppressing the phase information that it carries.

\section{SNR ESTIMATION BASED ON INFORMATION POTENTIAL}

Given equation (9) we can build an estimate of $S$ and $N$ for a given kernel bandwidth $v$. However, since (9) is undetermined, with two unknowns and one equation, and the entropy measure is sensitive to the scale, we need to combine this measure with another equation that allows us to solve the system of equations.

An initial solution is to use the second order moment. This transformation can be seen as equivalent to adding the equation $M_{2}=S+N$ to the system, which is then expressed as

$$
\left\{\begin{array}{ll}
V_{\mathcal{C}}\left(S, N+v_{1}\right)= & \hat{U}_{v_{1}}(\boldsymbol{x}) \\
S+N= & \hat{M}_{2}
\end{array} .\right.
$$


In this case we do not actually need to solve (11) but we can use $M_{2}$ to normalize the data first and then to measure the IP.

Consider an estimate of the second order moment, namely $\hat{M}_{2}$. The normalization is then

$$
x_{k}^{\prime}=\frac{x_{k}}{\sqrt{\hat{M}_{2}}}=\frac{x_{k}}{\sqrt{\frac{1}{K} \sum_{k=0}^{K-1}\left|x_{k}\right|^{2}}},
$$

which also normalizes the noise and signal powers

$$
\begin{aligned}
N^{\prime} & =N /(N+S) \\
S^{\prime} & =1-N^{\prime}=S /(N+S),
\end{aligned}
$$

and the SNR corresponds to

$$
\rho=\left(1-N^{\prime}\right) / N^{\prime} .
$$

Consequently, the estimator is based on solving the following equation:

$$
E\left\{\hat{U}_{v}\left(\boldsymbol{x}^{\prime}\right)\right\}=V_{\mathcal{C}}\left(1-N^{\prime}, N^{\prime}+v\right) .
$$

Since (15) is a nonlinear function, a root finding algorithm for approximating the solution $\hat{N}^{\prime}$ is used. For instance, the Newton method has a quadratic rate of convergence and takes only a few steps [2].

However, the function $V_{\mathcal{C}}\left(1-N^{\prime}, N^{\prime}+v\right)$ becomes a nonmonotonic function with respect to the SNR for any $v$, impeding a good functioning of the root finding algorithm. Figure 1 shows the function $V_{\mathcal{C}}\left(1-N^{\prime}, N^{\prime}+v\right)$ for multiple values of the kernel bandwidth of a 16-QAM constellation, with $v=0$ corresponding to the true IP value. The black line shows the asymptotic value of IP at high SNR, which can be seen merged with the true IP value around $15 \mathrm{~dB}$ of SNR. On the other side, the horizontal dotted line corresponds to the asymptotic value of the IP at very low SNR, which is $1 / 2 \pi$, and serves as a limit to show the curvature of the function under study. As a consequence, the root finding algorithm cannot find the proper solution if the actual SNR falls below the threshold value in which the IP is non-monotonic, which is actually different for each constellation. Although we can avoid this problem by focusing only at high SNR, it is impractical to implement at low and medium SNR values since it can result in two possible solutions. Consequently, an alternative is needed.

In order to avoid the problems associated with the nonmonotonic function, we will build a system of equations by measuring the IP for two different kernel bandwidth values, namely $v_{1}$ and $v_{2}$ :

$$
\left\{\begin{array}{lll}
V_{\mathcal{C}}\left(S, N+v_{1}\right) & = & \hat{U}_{v_{1}}(\boldsymbol{x}) \\
V_{\mathcal{C}}\left(S, N+v_{2}\right) & = & \hat{U}_{v_{2}}(\boldsymbol{x})
\end{array} .\right.
$$

Note that we do not need to normalize the data in order to build this estimator. Again, since (16) contains nonlinear functions, a root-finding algorithm for approximating the solution $\{\hat{N}, \hat{S}\}$ is needed. In this case the Newton method can also be used, but in its bidimensional form. Although building the system of equations lessens the previous problems, it is still difficult to retrieve the SNR from the estimation of IP around the

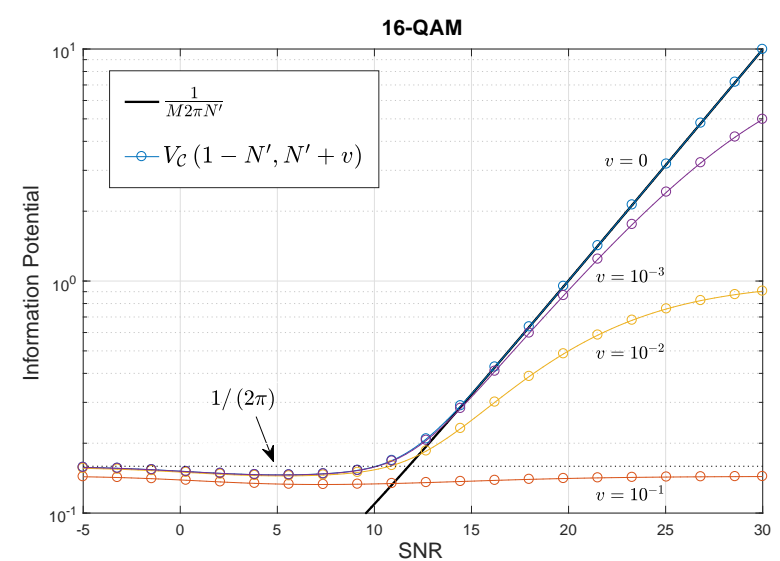

Fig. 1. Information potential of a 16-QAM constellation for several SNR values in $\mathrm{dB}$ evaluated with different kernel bandwidth $v$, jointly with the asymptotic value at high SNR that is $1 /(M 2 \pi N)$.

conflicting values of SNR, as we will see in the numerical results in Section IV.

Additionally, Figure 1 shows the IP for different values of kernel bandwidth $v$. By simple inspection the figure tells that the kernel bandwidth should become smaller as the SNR increases, and therefore $N^{\prime}$ decreases, in order to not induce bias in the estimate. This effect and the corresponding choice will be studied in the following subsection.

\section{A. Determining the kernel bandwidth}

Before determining the kernel bandwidth $v$, or $v_{1}$ when applicable, we must briefly review how the kernel estimator in (6) works, since the IP estimate greatly depends on $v$. Specifically, we would like to have a smaller as possible $v$ in order to reduce the bias, but doing so increases the variance. This trade-off is generally solved by applying a rule of thumb [12], which determines that the kernel bandwidth should be roughly around the variance of the data being processed. As a consequence, we want the values of kernel bandwidth around the noise power $N$. If we choose lower values of $v$ then the variance of the estimator is increased, and for higher values it tends to mask the original IP values. When using the estimator in (16) we want to make sure that $v_{1}$ is around the actual values of $N$, and $v_{2}$ just needs to be arbitrarily slightly higher than $v_{1}$.

In order to determine $v$, a first guess $v[0]$ is selected by an initial estimate of the IP for any reasonable kernel bandwidth (which can be, for instance, the $\hat{N}$ estimated by the $M_{2} M_{4}$ algorithm). Then, the new $v[q]$ is corrected following (8), and these steps are iterated until no significant changes are observed, a similar procedure used for the kernel bandwidth choice in [5]. Algorithm 1 depicts the iterative method for determining $v$. This algorithm actually takes very few iterations to converge, for a reasonable stopping rule $\delta$ around the order of $10^{-2}$, and this does not depend on the actual SNR value. Note that since the procedure is based on the high SNR approximation of the IP, the estimate of the kernel bandwidth will tend to the true value of noise power $N$ as the SNR increases and the kernel bandwidth decreases. This fact encourages to determine an estimate based on this algorithm only for high SNR values, which will be reviewed 


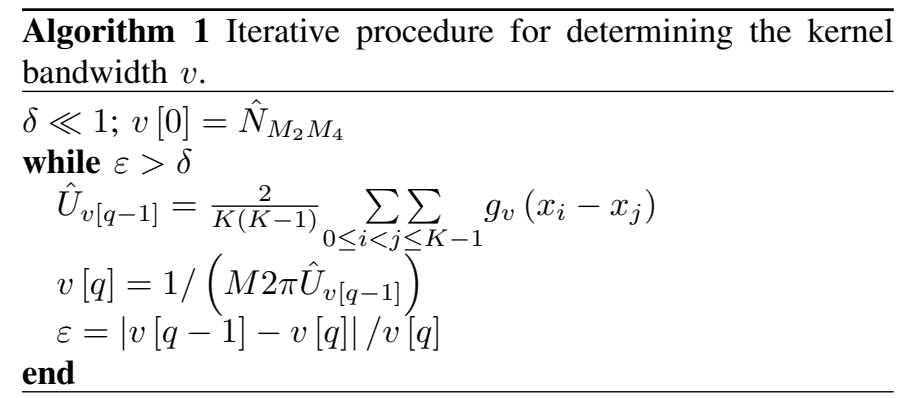

in the next subsection. On the other hand, at low and medium SNR values it provides a rough estimate of $N$, but convenient to determine $v_{1}$ and $v_{2}$ for the final estimator in (16).

\section{B. Determining the threshold and estimation at high SNR}

We have already stated that for high SNR values we can approximate the IP given in (8). If the conditions are met, the estimation of SNR becomes much simpler thanks to the one to one transformation provided by (8), thus avoiding the system solving step. However, we first need to define a threshold $\gamma$ that determines when the SNR is considered sufficiently high. While we could determine $\gamma$ based on a first estimate of the SNR and then by comparing to the true threshold for each constellation, similarly as looking at Figure 1, we will base it on actual IP measures. In fact, we are interested on determining the IP of the constellation when the components of the GMM do not overlap between them, and use it as a threshold in which we can assume that equation (8) holds true. The rationale behind is that we are actually determining at which SNR the communication system works with an acceptable error rate, which is classically defined by the union bound that depends on distances between symbols.

For the task of determining $\gamma$ it is advantageous to first normalize the data following (12). The purpose of the normalization is twofold: for any value of $N^{\prime}$ we know exactly $S^{\prime}$, allowing to easily measure the high SNR threshold, and it provides the necessary statistic to combine with an estimate of the IP for being insensitive to the scale. We want to make sure that the following rule is met

$$
\min _{i \neq j}\left|\sqrt{S^{\prime}} s_{i}-\sqrt{S^{\prime}} s_{j}\right|^{2}=\left(1-N^{\prime}\right) \min _{i \neq j}\left|s_{i}-s_{j}\right|^{2}>3 \frac{N^{\prime}}{2},
$$

which means that we respect a space that is three times the nominal noise of a Gaussian component for one of its dimensions. Therefore, the threshold $\gamma$ is determined by the IP such that

$V_{\mathcal{C}, \gamma}\left(1-N^{\prime}, N^{\prime}\right)=\left(M 2 \pi\left(\frac{2 \min _{i \neq j}\left|s_{i}-s_{j}\right|^{2}}{3+2 \min _{i \neq j}\left|s_{i}-s_{j}\right|^{2}}\right)\right)^{-1}$.

Finally, if a first measure of the IP is greater than $V_{\mathcal{C}, \gamma}$, we can ensure that we are at the high SNR values and therefore we can estimate the SNR by solving first

$$
\hat{N}^{\prime}=\frac{1}{M 2 \pi \hat{U}_{v}(\boldsymbol{x})}-v,
$$

which is corrected by the perturbation of $v$, and then by measuring $\hat{\rho}=\left(1-\hat{N}^{\prime}\right) / \hat{N}^{\prime}$.
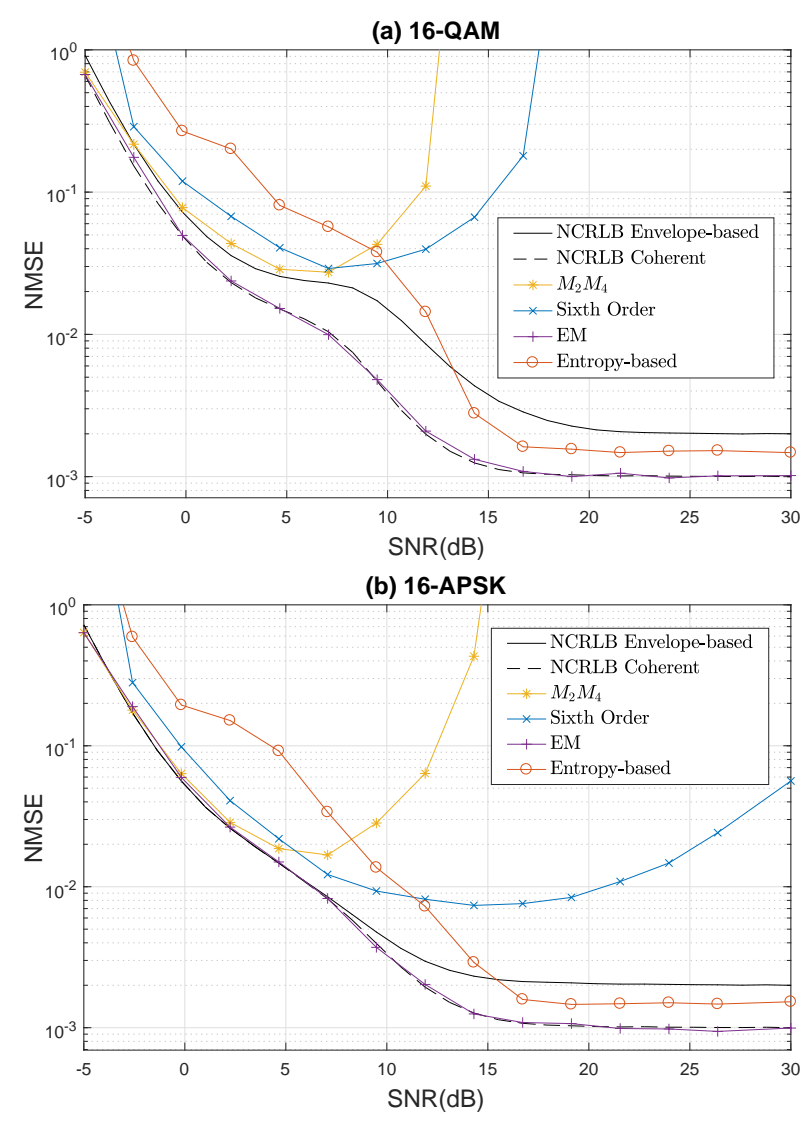

Fig. 2. Normalized mean square error of the estimated SNR for different estimators, jointly with both envelope-based and coherent CRLBs, with an observation window length of $K=1000$.

\section{NUMERIC RESULTS}

To illustrate the effectiveness of the proposed estimator, we will show the Normalized Mean Square Error (NMSE) of the estimated SNR with a frame length of $K=1000$ by means of Monte Carlo simulations. Each symbol for a given constellation was considered to be equally probable. The CRLB of the envelope-based and coherent schemes are measured following [8] and [7], respectively, and it will be compared with two moment-based methods $\left(M_{2} M_{4}\right.$ [10], and Sixth-order [9] for $b=2.75$ ) and the EM algorithm [4]. Note that while there are some envelope-based EM algorithms [8] we will compare the main results with a non envelopebased approach of the EM algorithm, thus providing a fair comparison. Finally, two different constellations are shown, 16-QAM and 16-APSK, in order to diversify different kind of number of rings and ring-ratios of the constellations and to show that it affects the moment-based algorithms but it does not affect the entropy-based one.

Figure 2 shows the aforementioned NMSE in terms of the actual value of SNR. We can see that at low and medium SNR values our algorithm struggles to reduce the variance, but it improves its performance as the SNR increases, particularly when the high SNR threshold in (18) is achieved. Note that while the moment-based method worsens at certain SNR values, it is around these values that our method improves, thus pointing to a possible collaboration algorithm in which the algorithm used is determined by the SNR range. Finally, while EM provides a 


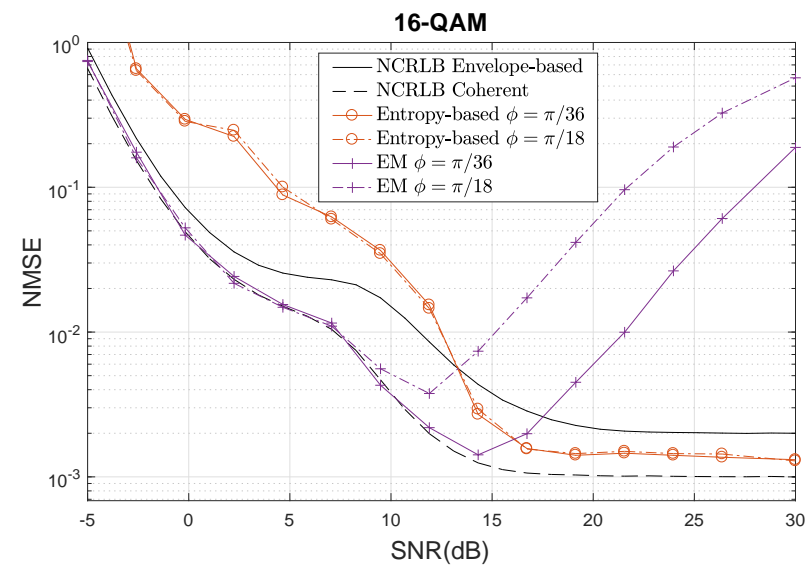

Fig. 3. Normalized mean square error of the estimated SNR for the entropybased and EM approaches for two different phase errors $\phi$, jointly with both envelope-based and coherent CRLBs, and the observation window length $K=$ 1000 .

consistent performance near the CRLB, it is very dependable on the initial conditions, which in this case is indicated by the SNR found by the $M_{2} M_{4}$ algorithm. Additionally, the EM algorithm has a higher computational complexity than the other approaches, particularly if convergence is not quickly achieved.

Figure 3 shows the same NMSE as the previous figure for a 16-QAM constellation, but with a phase error $\phi$ of $\pi / 36$ and $\pi / 18$ radians. The moment-based methods are not drawn since envelope-based algorithms are certainly invariant to phase errors, although the envelope-based CRLB is displayed for comparison. However, the entropy-based approach is shown for clarity while proving its invariance to rotations. In this case, if the phase error is not corrected, the EM algorithm deteriorates its performance for high SNR values, worsening for increasing values of $\phi$.

Lastly, figure 4 reviews the NMSE of the EM and entropybased estimators for an increasing value of $K$ without phase error. While the EM algorithm presents a gap in performance with respect to the entropy-based estimator, both estimators have a similar consistency for an increasing $K$. Additionally, multiple kernel bandwidths are shown, where each new kernel bandwidth $u$ is formed by scaling the $v$ given by Algorithm 1 . It is then shown the suitability of the bandwidth $v$ determined by the algorithm since both slightly increasing or decreasing this value harm the performance of the entropy-based estimator.

\section{CONCLUSIONS}

In this paper, we have proposed a novel entropy-based nondata-aided SNR estimator. The advantage is its invariance in front of phase errors and a reduced computational complexity compared to others non envelope-based algorithms. Simulations have shown that, although it struggles at low SNR, it can compete at high SNR with an improving performance with respect to the observation window length, closing distance with the EM algorithm but with computational complexity savings. Possible extensions of the current work involve the possibility of gaining robustness to frequency errors, wherein the observed data pairs in vicinity are more relevant than

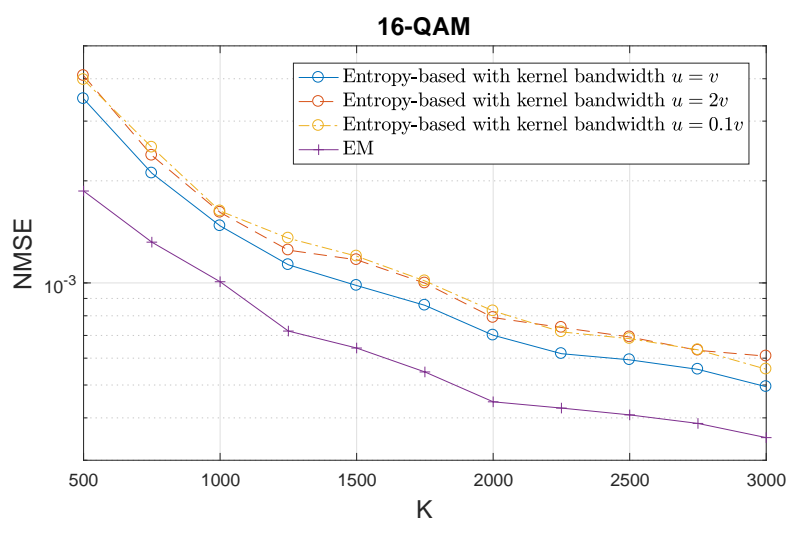

Fig. 4. Normalized mean square error of the estimated SNR for the entropybased and EM algorithms for an increasing observation window length $K$, $S N R=20 d B$, and three different kernel bandwidths $u$.

the ones far from each other in the kernel processing step. Additionally, it is of interest to study how the information potential can be extended to multiple-input multiple-output systems, allowing a more general definition of the problem.

\section{REFERENCES}

[1] M. Álvarez-Díaz, R. López-Valcarce, and C. Mosquera. SNR estimation for multilevel constellations using higher-order moments. IEEE Transactions on Signal Processing, 58(3):1515-1526, March 2010.

[2] A. Antoniou and W. Lu. Practical Optimization: Algorithms and Engineering Applications. Springer Publishing Company, 2007.

[3] T. R. Benedict and T. T. Soong. The joint estimation of signal and noise from the sum envelope. IEEE Transactions on Information Theory, IT13, 1967.

[4] A. Das. NDA SNR estimation: CRLBs and EM based estimators. In IEEE Region 10 Conf. TENCON, Hyderabad, India, pages 1-6, Nov 2008.

[5] F. de Cabrera, J. Riba, and G. Vázquez. Entropy-based covariance determinant estimation. In IEEE 18th International Workshop on Signal Processing Advances in Wireless Communications (SPAWC), 2017.

[6] Y. Fu, J. Zhu, S. Wang, and H. Zhai. Robust non-data-aided SNR estimation for multilevel constellations via Kolmogorov-Smirnov test. IEEE Communications Letters, 18(10):1707-1710, Oct 2014.

[7] W. Gappmair. Cramér-Rao lower bound for non-data-aided SNR estimation of linear modulation schemes. IEEE Transactions on Communications, 56(5), 2008.

[8] W. Gappmair, R. López-Valcarce, and C. Mosquera. Cramér-Rao lower bound and EM algorithm for envelope-based SNR estimation of nonconstant modulus constellations. IEEE Transactions on Communications, 57(6), 2009

[9] R. López-Valcarce and C. Mosquera. Sixth-order statistics-based nondata-aided SNR estimation. IEEE Communications Letters, 11(4):351353, April 2007.

[10] R. Matzner and F. Englberger. An SNR estimation algorithm using fourth-order moments. In Proceedings of 1994 IEEE International Symposium on Information Theory, June 1994.

[11] D. R. Pauluzzi and N. C. Beaulieu. A comparison of SNR estimation techniques for the AWGN channel. IEEE Transactions on Communications, 48(10):1681-1691, Oct 2000.

[12] J. C. Príncipe. Information theoretic learning: Rényi's entropy and kernel perspectives. NewYork: Springer, 2010.

[13] A. Rényi. On measures of entropy and information. In 4th Berkeley Symp. Prob. Theory Maths. Stat, Berkeley, CA, USA, pages 547-561, 1961.

[14] J. Riba, J. Villares, and G. Vázquez. A nondata-aided SNR estimation technique for multilevel modulations exploiting signal cyclostationarity. IEEE Transactions on Signal Processing, 58(11), 2010.

[15] E. Stathakis, J. Jaldén, L. K. Rasmussen, and M. Skoglund. Uniformly improving maximum-likelihood SNR estimation of known signals in Gaussian channels. IEEE Transactions on Signal Processing, 62(1):156167, Jan 2014. 